\title{
Effectiveness of targeted enhanced terminal room disinfection on hospital-wide acquisition and infection with multidrug-resistant organisms and Clostridium difficile: a secondary analysis of a multicentre cluster randomised controlled trial with crossover design (BETR Disinfection)
}

Deverick J Anderson, Rebekah W Moehring, David J Weber, Sarah S Lewis, Luke F Chen, J Conrad Schwab, Paul Becherer, Michael Blocker,

Patricia F Triplett, Lauren P Knelson, Yuliya Lokhnygina, William A Rutala, Daniel J Sexton, for the CDC Prevention Epicenters Program

\section{Summary}

Background The hospital environment is a source of pathogen transmission. The effect of enhanced disinfection strategies on the hospital-wide incidence of infection has not been investigated in a multicentre, randomised controlled trial. We aimed to assess the effectiveness of four disinfection strategies on hospital-wide incidence of multidrug-resistant organisms and Clostridium difficile in the Benefits of Enh anced Fr minal Bo m (BETR) Disinfection study.

Methods We did a prespecified secondary analysis of the results from the BETR Disinfection study, a pragmatic, multicentre, crossover cluster-randomised trial that assessed four different strategies for terminal room disinfection in nine hospitals in the southeastern USA. Rooms from which a patient with a specific infection or colonisation (due to the target organisms C difficile, meticillin-resistant Staphylococcus aureus, vancomycin-resistant enterococci (VRE), or multidrug-resistant Acinetobacter spp) was discharged were terminally disinfected with one of four strategies: standard disinfection (quaternary ammonium disinfectant, except for $C$ difficile, for which $10 \%$ hypochlorite [bleach] was used; reference); standard disinfection and disinfecting ultraviolet light (UV-C), except for $C$ difficile, for which bleach and UV-C was used (UV strategy); 10\% hypochlorite (bleach strategy); and bleach and UV-C (bleach and UV strategy). We randomly assigned the sequence of strategies for each hospital (1:1:1:1), and each strategy was used for 7 months, including a 1-month wash-in period and 6 months of data collection. The prespecified secondary outcomes were hospital-wide, hospital-acquired incidence of all target organisms (calculated as number of patients with hospital-acquired infection with a target organism per 10000 patient days), and hospital-wide, hospital-acquired incidence of each target organism separately. BETR Disinfection is registered with ClinicalTrials.gov, number NCT01579370.

Findings Between April, 2012, and July, 2014, there were 271740 unique patients with 375918 admissions 314610 admissions met all inclusion criteria $(n=73071$ in the reference study period, $n=81621$ in the UV study period, $n=78760$ in the bleach study period, and $n=81158$ in the bleach and UV study period). 2681 incidenct cases of hospitalacquired infection or colonisation occurred during the study. There was no significant difference in the hospital-wide risk of target organism acquisition between standard disinfection and the three enhanced terminal disinfection strategies for all target multidrug-resistant organisms (UV study period relative risk [RR] 0.89, 95\% CI 0.79-1.00; $\mathrm{p}=0.052$; bleach study period $0 \cdot 92,0 \cdot 79-1 \cdot 08 ; \mathrm{p}=0 \cdot 32$; bleach and UV study period $0 \cdot 99,0 \cdot 89-1 \cdot 11 ; \mathrm{p}=0 \cdot 89)$. The decrease in risk in the UV study period was driven by decreases in risk of acquisition of $C$ difficile (RR $0 \cdot 89,95 \% \mathrm{CI}$ $0 \cdot 80-0 \cdot 99 ; \mathrm{p}=0 \cdot 031)$ and VRE $(0 \cdot 56,0 \cdot 31-0 \cdot 996 ; \mathrm{p}=0 \cdot 048)$.

Interpretation Enhanced terminal room disinfection with UV in a targeted subset of high-risk rooms led to a decrease in hospital-wide incidence of $C$ difficile and VRE. Enhanced disinfection overcomes limitations of standard disinfection strategies and is a potential strategy to reduce the risk of acquisition of multidrug-resistant organisms and $C$ difficile.

Funding US Centers for Disease Control and Prevention.

\section{Introduction}

Health-care-associated infections kill 75000 patients each year in the USA. ${ }^{1}$ Multidrug-resistant organisms are common causes of these infections, with Clostridium difficile being the most common cause. ${ }^{2}$ Health-careassociated infections lead to adverse patient outcomes, including increased duration of hospital stay, morbidity, and mortality. ${ }^{3-5}$

\section{Lancet Infect Dis 2018; 18: 845-53 \\ Published Online June 4, 2018 http://dx.doi.org/10.1016/ S1473-3099(18)30278-0 \\ See Comment page 814}

Duke Center for Antimicrobial Stewardship and Infection Prevention, Department of Medicine, Duke University School of Medicine, Durham, NC, USA (D) Anderson MD, RW Moehring MD, S S Lewis MD, LFChen MBBS, LPKnelson MSPH, Prof D J Sexton MD); Durham Veterans Affairs Medical Center, Durham, NC, USA

(RW Moehring); Department of Hospital Epidemiology, University of North Carolina Health Care, Chapel Hill, NC, USA (Prof D J Weber MD, Prof W A Rutala PhD); High Point Regional Hospital, High Point, NC, USA (P F Triplett MD); Alamance Regional Medical Center, Burlington, NC, USA (M Blocker MD); Carolinas Medical Center, Charlotte, NC, USA (M Blocker); Rex Healthcare, Raleigh, NC USA (P Becherer MD), Chesapeake Regional Medical Center, Chesapeake, VA, USA (J C Schwab MD); and Department of Biostatistics and Bioinformatics, Duke University Medical Centre, Durham, NC, USA (Y Lokhnygina PhD) Correspondence to: Dr Deverick J Anderson, Duke Center for Antimicrobial Stewardship and Infection Prevention, Department of Medicine, Duke University School of Medicine, Durham, NC 27710, USA deverick.anderson@duke.edu 
Research in context

\section{Evidence before this study}

The hospital environment can be contaminated with multidrug-resistant organisms and is often disinfected inadequately. As a result, patients who enter contaminated hospital rooms are at increased risk for acquisition and infection with these multidrug-resistant organisms. Enhanced disinfection strategies might decrease the risk of transmission of multidrug-resistant organisms through the hospital environment, but supportive evidence is limited to single-centre, quasi-experimental studies. We searched PubMed using the terms "terminal disinfection" and "UV disinfection" to identify results of clinical studies published between Jan 1, 2003, and Jan 31, 2018. We identified 13 relevant studies, which were included in a meta-analysis that showed that UV disinfection was most effective at decreasing incidence of hospital-acquired Clostridium difficile and vancomycin-resistant enterococci (VRE). However, all but one of these studies were done in single institutions, with quasi-experimental study design, multiple interventions, and no appropriate controls.

The hospital environment is a source of pathogen transmission due to routine contamination with pathogens that can survive on inanimate surfaces for days or months..$^{6-8}$ Standard terminal disinfection, defined as the disinfection of a room after a patient's departure to prepare the room for a new occupant, does not sufficiently eliminate contamination. ' Consequently, patients admitted to rooms previously occupied by patients with multidrug-resistant organisms or $C$ difficile (so-called high-risk rooms) are at a substantially increased risk of subsequent infection or colonisation. ${ }^{10-13}$

In 2017, we reported the results of the Benefits of Enhanced Terminal Room (BETR) Disinfection study, the first multicentre randomised controlled trial to investigate strategies for enhanced terminal room disinfection. ${ }^{14}$ The results of this trial showed that adding ultraviolet light (UV-C) disinfection to standard chemical terminal disinfection of high-risk rooms led to a significant decrease in the risk of acquisition and infection with multidrug-resistant organisms and $C$ difficile for patients admitted to these rooms.

However, infectious outcomes in patients admitted to hospital are not independent; patient-level assessments will not fully capture the effect of enhanced disinfection strategies on the incidence of infections among all patients admitted to hospital. We aimed to assess the effect of the three enhanced terminal disinfection strategies investigated during the BETR Disinfection study on the hospital-wide incidence of $C$ difficile, meticillin-resistant Staphylococcus aureus (MRSA), vancomycin-resistant enterococci (VRE), and multidrugresistant Acinetobacter spp.

\section{Added value of this study}

To the best of our knowledge, this is the first multicentre, randomised controlled trial to investigate the effect of enhanced disinfection strategies on the hospital-wide incidence of hospital-acquired infections caused by four target multidrug-resistant organisms: C difficile, meticillin-resistant Staphylococcus aureus (MRSA), VRE, and multidrug-resistant Acinetobacter spp. The addition of a UV-C device to standard terminal room disinfection decreased the risk of subsequent acquisition and infection by target multidrug-resistant organisms such as C difficile and VRE. This study further shows that enhanced disinfection can have both a direct effect on the next patient who enters a contact isolation room and an indirect beneficial effect on other patients admitted to hospital.

\section{Implications of all the available evidence}

Infections with multidrug-resistant organisms lead to adverse outcomes. Novel prevention strategies are needed. Enhanced disinfection is a potential strategy to reduce the risk of acquisition of multidrug-resistant organisms, through both direct (for MRSA and VRE) and indirect effects ( $C$ difficile and VRE).

\section{Methods}

\section{Study design and participants}

We did a prespecified secondary analysis of the BETR Disinfection study, ${ }^{14}$ a pragmatic, prospective, multicentre, cluster-randomised trial with crossover design that assessed four different strategies for terminal room disinfection.

This study was designed to detect hospital-wide infection or colonisation due to one of four so-called target organisms: $C$ difficile, MRSA, VRE, or multidrugresistant Acinetobacter spp. ${ }^{15}$ We defined a history of infection or colonisation as any positive culture with a target organism within 12 months of admission. We defined community-onset infection as the isolation of a target organism within the first $48 \mathrm{~h}$ of hospital admission. We defined hospital-acquired as the isolation of a target organism after $48 \mathrm{~h}$ of hospitalisation. We defined a seed room as a room housing a patient with a microbiologically-proven current or past history of infection or colonisation with one or more target organisms.

In this study, we considered all patients admitted to a study hospital during the BETR study period (April, 2012, to July, 2014). Patients were included in the analysis if they were in the hospital for at least $48 \mathrm{~h}$. An incident case occurred during a hospital admission if the patient had a positive clinical culture or test with one of the target organisms. Patients were excluded if they had a microbiologically proven history of infection or colonisation with the same target organism during the 12 months previous to hospital admission, or had a community-onset infection. 
We considered all microbiological cultures for inclusion in our outcome analysis. Cultures could have been representative of infection or colonisation. We did not specifically obtain screening cultures for this study. All hospitals used nucleic acid amplification tests to identify $C$ difficile throughout the study.

The Duke University Health System institutional review board served as the central institutional review board. All participating hospitals received a waiver of informed consent and approval from their local institutional review boards.

\section{Randomisation and masking}

Each strategy was used at each study hospital during four sequential 7-month study periods. The sequence of strategies was randomly selected for each hospital, as described elsewhere. ${ }^{14}$ Each study period included a 1-month wash-in period, followed by a 6-month period of data collection. All hospitals used all four strategies in a 1:1:1:1 ratio. Allocation was not masked.

\section{Procedures}

All study hospitals employed gown and glove precautions (ie, contact precautions) for patients known or suspected to have colonisation or infection with target organisms. For the purpose of this study, these contact precaution rooms were defined as targeted rooms. Four strategies for terminal room disinfection were employed in targeted rooms. Strategy A, the reference period, involved the use of a quaternary ammonium-containing disinfectant (EnCompass Quaternary Disinfectant Cleaner and the EnCompass System, Ecolab, St Paul, MN, USA) in all targeted rooms except rooms of patients with $C$ difficile, in which a $10 \%$ hypochlorite-containing disinfectant (bleach; Clorox Germicidal Wipes, Clorox, Oakland, CA, USA) was used. Strategy B involved the use of a quaternary ammonium-containing disinfectant and a UV-C device in all targeted rooms except rooms of patients with $C$ difficile, in which a bleach-containing disinfectant and UV-C device were used. Strategy C involved the use of a bleach-containing disinfectant in all targeted rooms. Strategy D involved the use of a bleachcontaining disinfectant and a UV-C device for all targeted rooms. For ease of discussion, these four strategies will be labelled hereafter as reference (strategy A), UV (strategy B), bleach (strategy C), and bleach and UV (strategy D). All other rooms were considered to be nontargeted, and strategy A was used for terminal disinfection in non-targeted rooms throughout the study. Chemical disinfectants were standardised across study hospitals..$^{14}$

\section{Outcomes}

There were two prespecified secondary outcomes: hospital-wide, hospital-acquired incidence of all target organisms, and hospital-wide, hospital-acquired incidence of each target organism separately. We calculated hospital-wide incidence as the number of patients with hospital-acquired outcomes with a target organism per 10000 patient days among eligible patients.

\section{Statistical analysis}

Our a-priori hypothesis was that use of enhanced terminal disinfection in targeted rooms would lead to

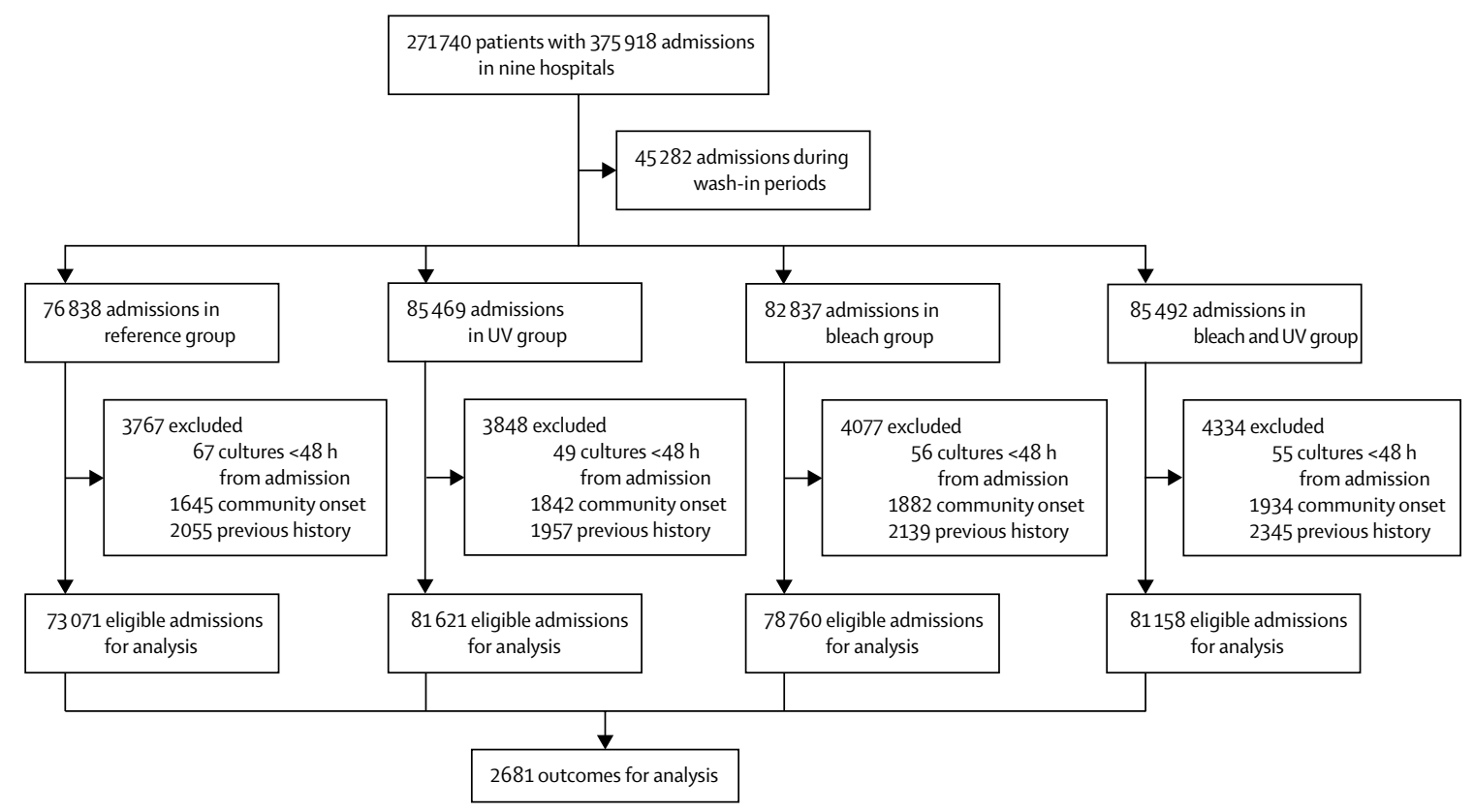


$\begin{array}{llll}\text { Standard disinfection period } & \text { UV period } & \text { Bleach period } & \text { Bleach and UV period } \\ \text { (reference group) }\end{array}$

\begin{tabular}{|c|c|c|c|c|}
\hline \multicolumn{5}{|l|}{ All target organisms } \\
\hline Exposed admissions & 73071 & 81621 & 78760 & 81158 \\
\hline Incident cases (\%) & $626(0.86 \%)$ & $683(0.84 \%)$ & $671(0.85 \%)$ & $701(0 \cdot 86 \%)$ \\
\hline Patient days & 345484 & 397222 & 382388 & 401822 \\
\hline Incidence (per 10000 patient days) & $18 \cdot 1$ & $17 \cdot 2$ & $17 \cdot 5$ & $17 \cdot 4$ \\
\hline Risk difference (95\% Cl) & 1 (ref) & $0.93(-0.83$ to 2.68$)$ & $0.57(-1.21$ to 2.35$)$ & $0.67(-1.12$ to 2.46$)$ \\
\hline Relative risk $(95 \% \mathrm{Cl})$; p value & 1 (ref) & 0.89 (0.79 to 1.00$) ; 0.052$ & 0.92 (0.79 to 1.08$) ; 0.32$ & 0.99 (0.89 to 1.11$) ; 0.89$ \\
\hline \multicolumn{5}{|l|}{ Clostridium difficile } \\
\hline Exposed admissions & 76099 & 84776 & 82193 & 84741 \\
\hline Incident cases (\%) & $375(0.49 \%)$ & $389(0.46 \%)$ & $362(0.44 \%)$ & $389(0.46 \%)$ \\
\hline Patient days & 372654 & 426157 & 411471 & 436330 \\
\hline Incidence (per 10000 patient days) & $10 \cdot 1$ & $9 \cdot 13$ & $8 \cdot 80$ & 8.92 \\
\hline Risk difference $(95 \% \mathrm{Cl})$ & 1 (ref) & $0.93(-0.31$ to 2.18$)$ & $1.27(0.005$ to 2.53$)$ & $1.15(-0.13$ to 2.43$)$ \\
\hline Relative risk $(95 \% \mathrm{Cl})$; p value & 1 (ref) & 0.89 ( 0.80 to 0.99$) ; 0.031$ & $0.91(0.75$ to 1.10$) ; 0.32$ & $0.97(0.84$ to 1.12$) ; 0.68$ \\
\hline \multicolumn{5}{|c|}{ Meticillin-resistant Staphylococcus aureus } \\
\hline Exposed admissions & 74273 & 82773 & 80008 & 82576 \\
\hline Incident cases (\%) & $204(0 \cdot 27 \%)$ & $259(0 \cdot 31 \%)$ & $234(0 \cdot 29 \%)$ & $242(0 \cdot 29 \%)$ \\
\hline Patient days & 360268 & 411857 & 397959 & 420338 \\
\hline Incidence (per 10000 patient days) & $5 \cdot 66$ & $6 \cdot 29$ & 5.88 & 5.76 \\
\hline Risk difference (95\% Cl) & 1 (ref) & $-0.63(-1.63$ to 0.37$)$ & $-0.22(-1.21$ to $0 \cdot 77)$ & $-0.10(-1.08$ to 0.89$)$ \\
\hline Relative risk $(95 \% \mathrm{Cl})$; p value & 1 (ref) & $1.08(0.89$ to 1.30$) ; 0.42$ & $0.97(0.76$ to 1.24$) ; 0.82$ & $1.00(0.87$ to 1.14$) ; 0.97$ \\
\hline \multicolumn{5}{|l|}{ Vancomycin-resistant enterococci } \\
\hline Exposed admissions & 76125 & 84733 & 81910 & 84466 \\
\hline Incident cases (\%) & $121(0 \cdot 16 \%)$ & $138(0 \cdot 16 \%)$ & $189(0.23 \%)$ & $194(0 \cdot 23 \%)$ \\
\hline Patient days & 373306 & 427099 & 409366 & 432599 \\
\hline Incidence (per 10000 patient days) & $3 \cdot 24$ & $3 \cdot 23$ & $4 \cdot 62$ & $4 \cdot 48$ \\
\hline Risk difference (95\% Cl) & 1 (ref) & 0.010 (-0.77 to 0.79$)$ & $-1 \cdot 38(-2 \cdot 21$ to -0.54$)$ & $-1.24(-2.06$ to -0.42$)$ \\
\hline Relative risk $(95 \% \mathrm{Cl})$; $\mathrm{p}$ value & 1 (ref) & $0.56(0.31$ to 0.996$) ; 0.048$ & 0.87 (0.65 to 1.17$) ; 0.35$ & 1.28 (0.94 to 1.73$) ; 0.11$ \\
\hline \multicolumn{5}{|l|}{ Multidrug-resistant Acinetobacter spp* } \\
\hline Exposed admissions & 62773 & 71840 & 68486 & 70249 \\
\hline Incident cases (\%) & $6(0.01 \%)$ & $3(0.00 \%)$ & $4(0.01 \%)$ & $3(0.00 \%)$ \\
\hline Patient days & 331711 & 382680 & 365424 & 388748 \\
\hline Rate (per 10000 patient days) & 0.18 & 0.08 & 0.11 & 0.08 \\
\hline Risk difference (95\% Cl) & 1 (ref) & $0.10(-0.07$ to 0.28$)$ & $0.07(-0.12$ to 0.26$)$ & $0.10(-0.07$ to 0.28$)$ \\
\hline
\end{tabular}

Table 1: Incidence of hospital acquisition of target multidrug-resistant organisms

decreases in the risk of hospital-wide, hospital-acquired target organisms for the hospital population. In other words, enhanced terminal room cleaning strategies in targeted rooms would have both direct and indirect effects, leading to decreased risk of acquisition of target organisms among all hospitalised patients.

We analysed incidence using overdispersed Poisson models, with disinfection strategy (reference, UV, bleach, and bleach and UV), order of the strategies within the study (whether a particular strategy was used in study period one, two, three, or four), and hospital as fixed-effect categorical covariates. We used generalised estimating equations to account for correlation between different study periods within the same hospital. Outcomes of patients admitted to hospital during each intervention were compared with the reference study period. The same model construction strategy was used for all outcomes. For each outcome, we calculated relative risk (RR), risk differences, and 95\% CIs. Statistical tests were done at a two-sided significance level of $0 \cdot 05$. We did no formal adjustments for multiple comparisons.

We also did a post-hoc analysis, to better assess if patients could receive indirect benefit from the use of UV for terminal disinfection. All study patients were separated into four categories, on the basis of the type of room entered and terminal room disinfection strategy used: (1) room cleaned with chemical disinfectants only and did not enter a confirmed seed room; (2) room cleaned with chemical disinfectants only and entered a 
confirmed seed room; (3) room cleaned with UV and did not enter a confirmed seed room; and (4) room cleaned with UV and entered a confirmed seed room.

We calculated incidence of each target pathogen in each category. We hypothesised that the use of UV in the subset of targeted rooms (categories 3 and 4) would lead to improved outcomes for other hospitalised patients (categories 1 and 2). We tested this hypothesis by comparing incidence among patients who did not enter a room disinfected with UV across study periods. We did not do statistical testing because of the post-hoc nature of this analysis.

Power estimates were based on review of 4 years of surveillance data from study hospitals and published literature. All power calculations were done with a two-sided 0.05 significance level. Our power calculation for this analysis was based on the following assumptions. After excluding the wash-in periods, we projected that 1.96 million patient days of care would be provided at the nine study hospitals, thus we projected that approximately 491200 patient days of care would occur for each 6-month study period. On the basis of data from our pre-existing surveillance databases, we projected that 959 outcomes due to the four target organisms would occur during the reference 6-month period, for a baseline incidence of health-care-associated infection of 19.5 per 10000 patient days. Under these assumptions, the study would have $60 \%$ power to detect a $10 \%$ decrease in the incidence rate, $92 \%$ power to detect a $15 \%$ decrease, and more than $99 \%$ power to detect a $20 \%$ decrease. We did the power analysis using simulation and it was based on a Poisson regression model with hospital-wide incidence rate as the outcome and study arm and hospital as the covariates. We did all statistical analyses using SAS (version 9.4).

BETR Disinfection is registered with ClinicalTrials.gov, number NCT01579370.

\section{Role of the funding source}

The funder of the study had no role in study design, data collection, data analysis, data interpretation, or writing of the report. The corresponding author had full access to all the data in the study and had final responsibility for the decision to submit for publication.

\section{Results}

All nine hospitals participated and were randomly assigned in the 28-month study from April, 2012, to July, 2014; none withdrew. A total of 271740 unique patients had 375918 admissions during the study (figure 1); 314610 admissions met all study criteria. A total of 2681 incident cases occurred during the study (table 1). During the reference period, 626 patients had an outcome during 345484 patient days (incidence $18 \cdot 1$ per 10000 patient days). The median incidence of target organisms per hospital was $17 \cdot 0$ per 10000 patient days (range 9.6-52.4) in the reference study period.

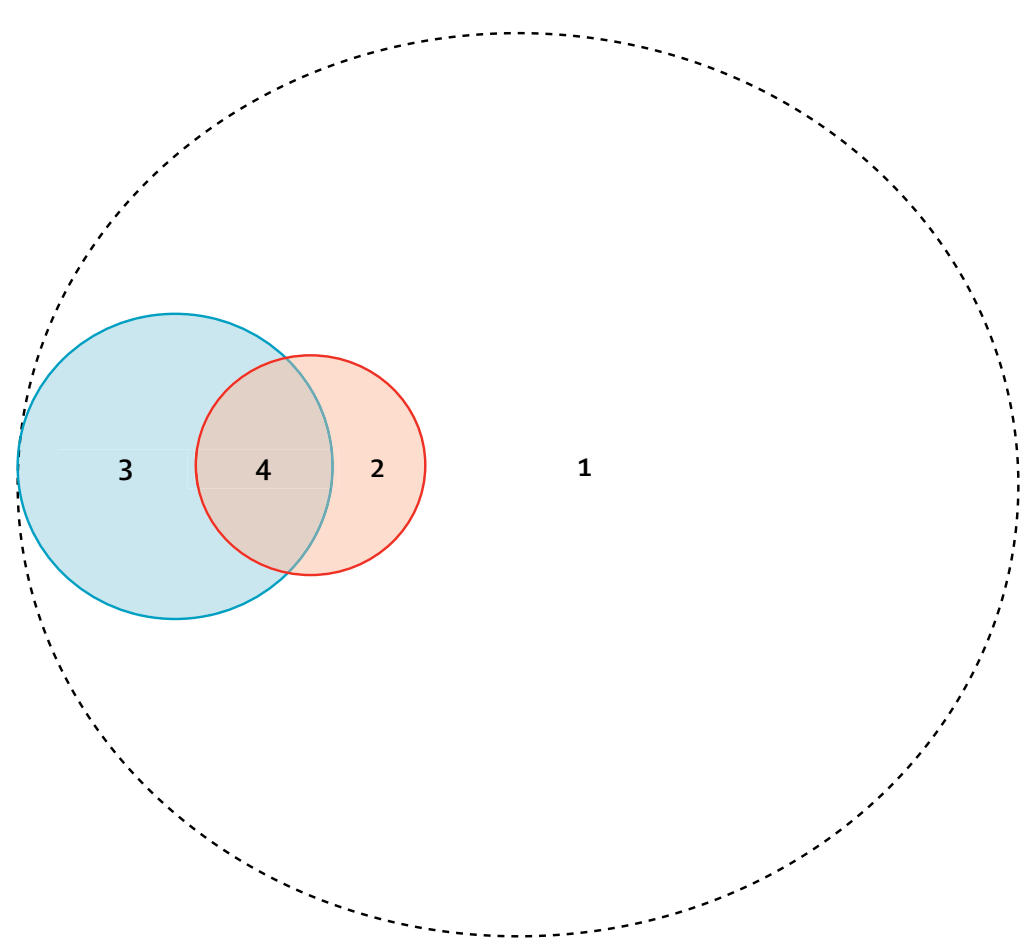

Figure 2: Four categories of exposure for patients admitted during the BETR Disinfection study

(1) Room cleaned with chemical disinfectants only and patient did not enter a confirmed seed room. (2) Room cleaned with chemical disinfectants only and patient entered into a confirmed seed room. (3) Room cleaned with UV and patient did not enter a confirmed seed room. (4) Room cleaned with UV and patient entered into a confirmed seed room. All patients admitted to hospital are included within the dashed circle. The size of the circles approximates the relative proportions of each category. The orange circle represents confirmed seed rooms. The blue circle represents rooms in which ultraviolet light was used during terminal disinfection.

Overall, there was no significant difference between standard disinfection and the three enhanced terminal disinfection strategies in the hospital-wide risk of target organism acquisition for all multidrug resistant organisms (table 1). The largest decrease in risk was in the UV study period $(n=683)$ compared with the reference study period $(\mathrm{n}=626$; incidence $17 \cdot 2$ per 10000 patient days vs $18 \cdot 1$ per 10000 patient days; RR $0 \cdot 89,95 \%$ CI $0 \cdot 79-1 \cdot 00$; $\mathrm{p}=0 \cdot 052$; table 1).The use of bleach in targeted rooms $(n=671)$ did not significantly decrease risk, compared with standard disinfection (incidence 17.5 per 10000 patient days vs $18 \cdot 1$ per 10000 patient days; $0 \cdot 92,0 \cdot 79-1 \cdot 08 ; \mathrm{p}=0 \cdot 32$ ). Hospital-wide risk of target organism acquisition was similar in both the bleach and UV $(n=701)$ and the reference study periods $(17.4$ per 10000 patient days vs $18 \cdot 1$ per 10000 patient days; $0 \cdot 99,0 \cdot 89-1 \cdot 11 ; \mathrm{p}=0 \cdot 89$ ).

The hospital-wide risk of $C$ difficile was significantly lower in the UV study period (rate 9.13 per 10000 patient days vs $10 \cdot 1$ per 10000 patient days; RR $0 \cdot 89$, 95\% CI $0 \cdot 80-0 \cdot 99$; $\mathrm{p}=0.031$ ), but was not significantly lower in the bleach study period (8.80 per 10000 patient days; $0.91,95 \% \mathrm{CI}$ $0.75-1.10 ; \mathrm{p}=0 \cdot 32$ ) or bleach and UV study period (8.92 per 10000 patient days; $0 \cdot 97,0 \cdot 84-1 \cdot 12$; $\mathrm{p}=0 \cdot 68$ ). The risk of MRSA was essentially unchanged in all study periods and increased in the UV study period (table 1). The 
risk of VRE was significantly lower in the UV study period than in the reference study period (incidence 3.23 per 10000 patient days vs 3.24 per 10000 patient days; $0 \cdot 56,0 \cdot 31-0 \cdot 99 ; \mathrm{p}=0 \cdot 048)$. The use of bleach led to a nonsignificant decrease in the risk of VRE whereas the risk was higher than reference in the bleach and UV study period.

Admissions were grouped into four exposure categories for our post-hoc analysis (figure 2; table 2). Patients who

Standard UV period $\quad$ Bleach period Bleach and period
disinfection period
(reference group)

\begin{tabular}{lllll}
\hline $\begin{array}{l}\text { Clostridium difficile } \\
\text { Exposure category } 1\end{array}$ & Quat & Quat & Quat & Quat \\
Exposure category 2 & Bleach & Bleach & Bleach & Bleach \\
Exposure category 3* & NA & Quat and UV-C & NA & Bleach and UV-C \\
Exposure category 4* & NA & Bleach and UV-C & NA & Bleach and UV-C \\
Vegetative bacteria (MRSA, VRE, Acinetobacter spp) & & \\
Exposure category 1 & Quat & Quat & Quat & Quat \\
Exposure category 2 & Quat & Quat & Bleach & Bleach \\
Exposure category 3* & NA & Quat and UV-C & NA & Bleach and UV-C \\
Exposure category 4* & NA & Quat and UV-C & NA & Bleach and UV-C
\end{tabular}

Quat=quaternary ammonium-containing disinfectant. UV-C=disinfecting ultraviolet light. MRSA=meticillin-resistant Staphylococcus aureus. VRE=vancomycin-resistant enterococci. *Only relevant for UV study groups.

Table 2: Differences in terminal disinfection strategies for each exposure category entered rooms that were cleaned using standard disinfection strategies (exposure categories 1 and 2) had lower incidence of $C$ difficile infection during UV study periods than in other study periods (table 3); 729 cases of hospital-acquired $C$ difficile infection occurred during 779049 patient days in non-UV study periods (incidence 9.36 per 10000 patient days) compared with 592 cases during 739048 patient days in UV study periods (incidence 8.01 per 10000 patient days). Incidence of hospital-acquired $C$ difficile was lower among patients in categories 1 and 2 during all three enhanced room disinfection strategies, compared with patients in categories 1 and 2 in the reference study period (table 3 ).

Among patients not exposed to rooms cleaned with UV, patients admitted to seed rooms (category 2) had approximately double or higher the incidence of patients admitted to non-seed rooms (category 1) for MRSA and VRE, and for most exposure categories (table 3). The incidence of hospital-acquired VRE after admission to a VRE seed room was five-times to ten-times higher than the incidence for patients not admitted to a VRE seed room.

\section{Discussion}

For all target organisms, the use of enhanced terminal room disinfection strategies in targeted, high-risk rooms led to a non-significant hospital-wide decrease in the risk

All patients in categories 1 and 2 Did not enter a seed room (category 1) Entered a seed room (category 2)

\section{Clostridium difficile}

Non-UV disinfection strategy groups

UV disinfection strategy groups

Individual disinfection strategy groups

Reference

UV

Bleach

Bleach and UV

Meticillin-resistant Staphylococcus aureus

Non-UV disinfection strategy groups

UV disinfection strategy groups

Individual disinfection strategy groups

Reference

UV

Bleach

Bleach and UV

Vancomycin-resistant enterococci

Non-UV disinfection strategy groups

UV disinfection strategy groups

Individual disinfection strategy groups

Reference

UV

Bleach

Bleach and UV
729/779 049; $9 \cdot 36$

592/739048; 8.01

$372 / 369737 ; 10 \cdot 1$

303/370 199; 8.18

$357 / 409312 ; 8.72$

$289 / 368849 ; 7 \cdot 83$

$434 / 753385 ; 5 \cdot 76$

$394 / 716204 ; 5 \cdot 50$

204/357479; $5 \cdot 71$

$208 / 358995 ; 5.79$

$230 / 395906 ; 5.81$

$186 / 357209 ; 5 \cdot 21$

$304 / 777649 ; 3.91$

$208 / 739366 ; 2 \cdot 81$

$119 / 370344 ; 3 \cdot 21$

$89 / 371767 ; 2 \cdot 39$

$185 / 407305 ; 4.54$

$119 / 367599 ; 3.24$

\section{5/757193; 9.18}

583/730 619; 7.98

$353 / 358875 ; 9 \cdot 84$

296/365100; 8.11

$342 / 398318 ; 8.59$

287/365 519; $7 \cdot 85$

$365 / 690566 ; 5 \cdot 29$

$360 / 687624 ; 5 \cdot 24$

$171 / 327342 ; 5 \cdot 22$

$191 / 344721 ; 5 \cdot 54$

$194 / 363224 ; 5 \cdot 34$

169/342 903; 4.93

235/750260; $3 \cdot 13$

$188 / 728617 ; 2.58$

$96 / 358867 ; 2.68$

$79 / 367108 ; 2.15$

139/391393; 3.55

109/361509; 3.02
$34 / 21856 ; 15 \cdot 6$

$9 / 8429 ; 10 \cdot 7$

19/10862; 17.5

7/5099; 13.7

$15 / 10994 ; 13.6$

2/3330; 6.01

69/62 819; 11.0

$34 / 28580 ; 11.9$

33/30137; 11.0

$17 / 14274 ; 11.9$

$36 / 32682 ; 11.0$

$17 / 14306 ; 11.9$

69/27389; $25 \cdot 2$

20/10749; 18.6

23/11477; $20 \cdot 0$

$10 / 4659 ; 21 \cdot 5$

46/15 912; 28.9

10/6090; $16 \cdot 4$ 
of hospital-acquired $C$ difficile, MR SA, VR E, an d multidrug-resistant Acinetobacter spp. Although these findings were not significant, they supplement the primary results from our BETR Disinfection study, and provide further validity of the benefit of using enhanced terminal room disinfection methods. Our findings also show that enhanced disinfection with UV has both a direct effect on the next patient who enters a contact isolation room, ${ }^{14}$ and an indirect beneficial effect on other hospitalised patients. In particular, the addition of UV to standard chemical disinfection led to a significant reduction in the hospital-wide risk of acquiring $C$ difficile and VRE.

We believe enhanced terminal room disinfection strategies that target high-risk rooms led to a decrease in hospital-wide incidence of multidrug-resistant organisms and $C$ difficile th rough se veral $\mathrm{pl}$ ausible di rect an $\mathrm{d}$ indirect methods.

First, patients with one type of multidrug-resistant organism are often colonised with other multidrugresistant organisms. ${ }^{16}$ Thus, an intervention targeting one specific organism might concurrently decrease the risk for colonisation or infection with others.

Second, rooms of patients colonised or infected with one or more target organisms could serve as the epicentres for transmission within a hospital when contamination of shared equipment and health-care professionals' hands and clothing occurs. The hands and clothing of health-care professionals regularly become contaminated by both the patient and the environment. ${ }^{17-19}$

Finally, our strategy of using enhanced terminal room disinfection strategies in targeted, contact precaution rooms actually led to more uses of the UV-C device than was required for patients qualifying for assessment of the primary outcome in the BETR Disinfection study. ${ }^{14}$ We used the UV device 21844 times during the study, yet only 6549 (30\%) of these uses were in patients qualifying for the trial's primary outcome. This discrepancy can be explained by the difference in criteria provided to environmental services for conduct of the study (ie, any contact precautions room) and the strict study definitions that led to our definition of exposed for the primary outcome (ie, seed rooms, which relied on microbiological data). This approach led to the use of UV-C devices in non-seed rooms in numerous different ways. First, more than 5000 patients admitted to seed rooms were excluded from the primary analysis because they met one of our exclusion criteria. However, our study protocol dictated that a UV-C device would have been used in these rooms. Second, numerous patients were routinely placed in contact precautions because of a remote ( $>12$ month) history of infection or colonisation with one of our target multidrug-resistant organisms. Third, some patients were placed on contact precautions for other clinically important organisms (eg, norovirus, multidrug-resistant Gram-negative rods). Fourth, some patients were placed on contact precautions because they met criteria for syndromic surveillance. Finally, we know from discussions with local environmental services personnel that the UV-C devices were occasionally used by request of floor nurses. In summary, the inclusion of these additional rooms and the subsequent decrease in environmental bioburden of target organisms in contact precaution rooms probably contributed to our hospitalwide outcomes.

In light of the above discussion, we believe enhanced room disinfection interventions that decrease the overall cumulative burden of baseline environmental contamination in a hospital result in a secondary, indirect decrease in the hospital-wide incidence of hospitalacquired multidrug-resistant organisms. For example, once present in a room, these pathogens might serve as sources for subsequent exposure to multiple patients sequentially admitted in the same room or adjacent rooms over many days and even weeks. Investigators recently published a metagenomic analysis ${ }^{20}$ of more than 6000 samples from hospital surfaces, patients, and staff during a 12-month period surrounding the opening of a new hospital in Chicago. Patients routinely acquired roomassociated taxa during the first $24 \mathrm{~h}$ in the hospital room. Thus, changes that affect the hospital microbiome would logically affect the risk of patient exposure, particularly for organisms that might be part of the so-called faecal patina in hospitals, such as C difficile and VRE. ${ }^{21}$

The decreases in hospital-wide incidence observed in this study are consistent with previously published data on the use of UV-C devices. Authors of a meta-analysis of 13 studies $^{22}$ concluded that UV disinfection was most effective at decreasing incidence of hospital-acquired $C$ difficile and VRE. However, all but one of these studies (our primary analysis study) were done in single centres using quasi-experimental study design, included multiple interventions, did not have appropriate controls, and did not measure compliance with hand hygiene, measure environmental cleaning, or account for infinite time bias. By contrast, our study is the first multicentre, randomised controlled trial to show a decrease in hospital-wide incidence after implementation of enhanced terminal disinfection with UV. It remains unclear, however, why this effect was observed in the UV study period but not in the bleach and UV study period. It is conceivable that the microfibre used in the UV study period, implementation issues, or both, could have contributed to the difference. However, we note that the difference between the UV study period and bleach and UV study period is consistent throughout our results, including the patient-specific outcomes, microbiological outcomes, and hospital-wide outcomes.

Our findings are consistent with previously published data that patients admitted to rooms recently vacated by patients with multidrug-resistant organisms are at increased risk of infection. ${ }^{13}$ This finding further suggests that patients with asymptomatic colonisation with multidrug-resistant organisms might serve as a source of 
environmental contamination that increases risk for subsequent patients admitted to hospital. Coupled with implementation data from our trial, ${ }^{23}$ this finding also underscores the challenge of providing enhanced terminal disinfection with UV to high-risk rooms. Despite casting a wide net to capture eligible rooms, we only used the UV machine in $60 \%$ of seed rooms (see categories 2 and 4 in figure 2) because of delays in assessment of microbiological results or missed opportunities. Strategies to identify colonised patients more rapidly, finding methods to better implement enhanced terminal room disinfection strategies, and applying these strategies more globally could help overcome these limitations.

Adding enhanced disinfection with UV to standard chemical disinfection led to a decrease in hospital-wide acquisition of multidrug-resistant organisms and $C$ difficile, but this decrease was modest. Indeed, the effect of environmental disinfection on the incidence of $C$ difficile in particular might be modest. Ray and colleagues $^{24}$ recently published data from a 12-month randomised controlled trial involving 15 hospitals to investigate the effect of enhanced chemical disinfection with bleach on the incidence of hospital-onset $C$ difficile infection. ${ }^{24}$ Despite significantly improving cleaning compliance with bleach, the intervention did not lead to a decrease in the incidence of hospital-wide hospital-onset $C$ difficile. Results from our study are similar, because enhanced use of bleach did not decrease the hospitalwide incidence of hospital-onset $C$ difficile. Other published data also suggest that the role of the hospital environment on transmission of $C$ difficile might not be large. ${ }^{25-27}$ Although our data provide evidence that disinfection does indeed have some role to play in prevention, findings from these studies collectively show that hospitals need to employ additional strategies to specifically affect transmission of $C$ difficile. For example, hospitals should use antimicrobial stewardship teams to decrease the use of unnecessary antibiotics both in inpatient and outpatient settings, particularly by decreasing the use of high-risk antibiotics such as clindamycin and fluoroquinolones. ${ }^{27,28}$

Limitations of this study are similar to the limitations of our primary analysis. ${ }^{14}$ For example, we did not specifically screen patients for colonisation, but relied on cultures obtained during routine clinical practice. Second, we did not perform formal adjustments for multiple testing. Third, we did not measure antimicrobial use during our study. Therefore, the decrease observed with $C$ difficile could be related to changes in antimicrobial administration during the study. This seems unlikely, however, given the randomised nature of the study. Fourth, our implementation strategies, outlined in detail elsewhere ${ }^{23}$ might not be feasible in all hospital settings. Fifth, our study was performed in hospitals in the southeastern USA. Although our results included data from both tertiary and community hospitals, care provided in study settings might not be generalisable to all locations. Finally, our hospital-wide analyses were limited to four organisms and outcomes that occurred while the patient remained in hospital. Thus, the effects observed in our study might represent the minimum decrease achievable from enhanced disinfection strategies.

In conclusion, enhanced terminal room disinfection with UV in a targeted subset of high-risk rooms (ie, contact precaution rooms) led to a decrease in risk of acquisition of target multidrug-resistant organisms such as $C$ difficile and VRE for all hospitalised patients, through both direct and indirect effects. These findings are important, because they suggest that strategies targeting high-risk rooms might have benefit for the larger population of patients admitted to hospital by reducing the burden of pathogenic organisms in the hospital microbiome. The struggle against the transmission of and infections from multidrug-resistant organisms and $C$ difficile requires diligence, effort, and evidence-based strategies. Data from our study should help expand our understanding of the importance of disinfection practices in the hospital setting.

\section{Contributors}

All authors had full access to all of the data in the study and take responsibility for the integrity of the data and the accuracy of the data analysis. DJA, RWM, DJW, SSL, LFC, JCS, PB, MB, PFT, LPK, and WAR collected data for the study. DJA, RWM, DJW, SSL, LFC, JCS, PB, MB, PFT, LPK, WAR, and DJS assisted with data interpretation. DJA and YL developed the study design and created figures for the study. DJA and YL did the analyses. DJA did the literature search and wrote the first draft of the paper. DJS was responsible for funding for the study. All authors assisted with the writing process.

\section{Declaration of interests}

DJW and WAR report participation on advisory panels for Clorox, Inc. All other authors declare that we have no conflicts of interest.

\section{Acknowledgments}

DJS was supported by a grant from the Centers for Disease Control and Prevention (CDC) Epicenters Program (U54CK000164) and DJA was supported by a grant from the National Institutes of Health/Nationa Institute of Allergy and Infectious Diseases (NIH/NIAID; K23AI095357) The views expressed in this article are those of the authors and do not necessarily represent the views of the CDC or NIH.

The authors are deeply indebted to the infection control teams, information technology personnel, and environmental services staff and supervisors at each of the nine study hospitals. We thank these wonderful people for their contributions to the successful completion of this study. The authors would also like to acknowledge the contributions to the study made by TruD SmartUVC, Ecolab Inc, and The Clorox Company for their significant material contributions to the study. Additionally, the authors would like to thank Angelica Corporation and Shared Linen Services, each for donating microfibre cloths to a study hospital. All of these companies provided materials for the completion of the study but played no role in the funding, design, analysis, or manuscript preparation.

\section{References}

1 Magill SS, Edwards JR, Bamberg W, et al. Multistate point-prevalence survey of health care-associated infections. N Engl J Med 2014; 370: 1198-208.

2 Miller BA, Chen LF, Sexton DJ, Anderson DJ. Comparison of the burdens of hospital-onset, healthcare facility-associated Clostridium difficile infection and of healthcare-associated infection due to methicillin-resistant Staphylococcus aureus in community hospitals. Infect Control Hosp Epidemiol 2011; 32: 387-90. 
3 Cosgrove SE, Sakoulas G, Perencevich EN, Schwaber MJ, Karchmer AW, Carmeli Y. Comparison of mortality associated with methicillin-resistant and methicillin-susceptible Staphylococcus aureus bacteremia: a meta-analysis. Clin Infect Dis 2003; 36: 53-59.

4 DiazGranados CA, Jernigan JA. Impact of vancomycin resistance on mortality among patients with neutropenia and enterococcal bloodstream infection. J Infect Dis 2005; 191: 588-95.

5 Dubberke ER, Butler AM, Reske KA, et al. Attributable outcomes of endemic Clostridium difficile-associated disease in nonsurgical patients. Emerg Infect Dis 2008; 14: 1031-38.

6 Neely AN, Maley MP. Survival of enterococci and staphylococci on hospital fabrics and plastic. J Clin Microbiol 2000; 38: 724-26.

7 Weber DJ, Rutala WA. Role of environmental contamination in the transmission of vancomycin-resistant enterococci. Infect Control Hosp Epidemiol 1997; 18: 306-09.

8 Catalano M, Quelle LS, Jeric PE, Di Martino A, Maimone SM. Survival of Acinetobacter baumannii on bed rails during an outbreak and during sporadic cases. J Hosp Infect 1999; 42: 27-35.

9 Carling PC, Parry MM, Rupp ME, Po JL, Dick B, Von Beheren S. Improving cleaning of the environment surrounding patients in 36 acute care hospitals. Infect Control Hosp Epidemiol 2008; 29: $1035-41$.

10 Huang SS, Datta R, Platt R. Risk of acquiring antibiotic-resistant bacteria from prior room occupants. Arch Intern Med 2006; 166: 1945-51.

11 Shaughnessy MK, Micielli RL, DePestel DD, et al. Evaluation of hospital room assignment and acquisition of Clostridium difficile infection. Infect Control Hosp Epidemiol 2011; 32: 201-06.

12 Drees M, Snydman DR, Schmid CH, et al. Prior environmental contamination increases the risk of acquisition of vancomycin-resistant enterococci. Clin Infect Dis 2008; 46: 678-85.

13 Mitchell BG, Dancer SJ, Anderson M, Dehn E. Risk of organism acquisition from prior room occupants: a systematic review and meta-analysis. J Hosp Infect 2015; 91: 211-17.

14 Anderson DJ, Chen LF, Weber DJ, et al. Enhanced terminal room disinfection and acquisition and infection caused by multidrug-resistant organisms and Clostridium difficile (the Benefits of Enhanced Terminal Room Disinfection study): a cluster-randomised, multicentre, crossover study. Lancet 2017; 389: 805-14.

15 Magiorakos AP, Srinivasan A, Carey RB, et al. Multidrug-resistant, extensively drug-resistant and pandrug-resistant bacteria: an international expert proposal for interim standard definitions for acquired resistance. Clin Microbiol Infect 2012; 18: 268-81.

16 Donskey CJ. The role of the intestinal tract as a reservoir and source for transmission of nosocomial pathogens. Clin Infect Dis 2004; 39: 219-26.
17 Burden M, Cervantes L, Weed D, Keniston A, Price CS, Albert RK. Newly cleaned physician uniforms and infrequently washed white coats have similar rates of bacterial contamination after an 8-hour workday: a randomized controlled trial. J Hosp Med 2011; 6: 177-82.

18 Treakle AM, Thom KA, Furuno JP, Strauss SM, Harris AD Perencevich EN. Bacterial contamination of health care workers' white coats. Am J Infect Control 2009; 37: 101-05.

19 Anderson DJ, Addison R, Lokhnygina Y, et al. The antimicrobial scrub contamination and transmission (ASCOT) trial: a three-arm, blinded, randomized controlled trial with crossover design to determine the efficacy of antimicrobial-impregnated scrubs in preventing healthcare provider contamination. Infect Control Hosp Epidemiol 2017; 38: 1147-54.

20 Lax S, Sangwan N, Smith D, et al. Bacterial colonization and succession in a newly opened hospital. Sci Transl Med 2017; published online May 24. DOI:10.1126/scitranslmed.aah6500.

21 Weinstein RA. Intensive care unit environments and the fecal patina: a simple problem? Crit Cre Med 2012; 40: 1333-34.

22 Marra AR, Schweizer ML, Edmond MB. No-touch disinfection methods to decrease multidrug-resistant organism infections: a systematic review and meta-analysis Infect Control Hosp Epidemiol 2018; 39: 20-31.

23 Anderson DJ, Knelson LP, Chen LF, et al. Implementation lessons learned from the Benefits of Enhanced Terminal Room (BETR) Disinfection trial: process and perceptions of enhanced disinfection with UV devices. Infect Control Hosp Epidemiol 2018; 39: 157-63.

24 Ray AJ, Deshpande A, Fertelli D, et al. A multicenter randomized trial to determine the effect of an environmental disinfection intervention on the incidence of healthcare-associated Clostridium difficile infection. Infect Control Hosp Epidemiol 2017; 38: 777-83.

25 Eyre DW, Cule ML, Wilson DJ, et al. Diverse sources of $C$ difficile infection identified on whole-genome sequencing. N Engl J Med 2013; 369: 1195-205.

26 Widmer AF, Frei R, Erb S, et al. Transmissibility of Clostridium difficile without contact isolation: results from a prospective observational study with 451 patients. Clin Infect Dis 2017; 64: $393-400$

27 Dingle KE, Didelot X, Quan TP, et al. Effects of control interventions on Clostridium difficile infection in England: an observational study. Lancet Infect Dis 2017; 17: 411-21.

28 Freedberg DE, Salmasian H, Cohen B, Abrams JA, Larson EL Receipt of antibiotics in hospitalized patients and risk for Clostridium diffcile infection in subsequent patients who occupy the same bed. JAMA Intern Med 2016; 176: 1801-08. 